\title{
Central engines of Gamma Ray Bursts. Magnetic mechanism in the collapsar model.
}

\author{
Maxim V. Barkov*, and Serguei S. Komissarov* \\ ${ }^{*}$ Department of Applied Mathematics, The University of Leeds, Leeds, LS2 9GT,UK \\ ${ }^{\dagger}$ Space Research Institute, 84/32 Profsoyuznaya Street, Moscow 117997, Russia
}

\begin{abstract}
In this study we explore the magnetic mechanism of hypernovae and relativistic jets of long duration gamma ray bursts within the collapsar scenario. This is an extension of our earlier work[1]. We track the collapse of massive rotating stars onto a rotating central black hole using axisymmetric general relativistic magnetohydrodynamic code that utilizes a realistic equation of state and takes into account the cooling associated with emission of neutrinos and the energy losses due to dissociation of nuclei. The neutrino heating is not included. We describe solutions with different black hole rotation, mass accretion rate, and strength of progenitor's magnetic field. Some of them exhibits strong explosions driven by Poyntingdominated jets with power up to $12 \times 10^{51} \mathrm{erg} \mathrm{s}^{-1}$. These jets originate from the black hole and powered via the BlandfordZnajek mechanism. A provisional criterion for explosion is derived. A number of simulation movies can be downloaded from http://www.maths.leeds.ac.uk/ serguei/research/movies/anim.html
\end{abstract}

Keywords: black hole physics - supernovae: general - gamma-rays: bursts - methods: numerical - MHD - general relativity PACS: $95.30 . \mathrm{Qd} ;$ 97.10.Gz; 97.60.Lf; 97.60.Bw

\section{INTRODUCTION}

The most popular model of central engines of longsoft Gamma Ray Burst (GRB) is based on the "failed supernova" scenario of stellar collapse, or "collapsar", where the core of a rapidly rotating progenitor star forms a black hole $(\mathrm{BH})$ surrounded by an accretion disk [2]. A similar configuration can be produced via inspiraling of a $\mathrm{BH}$ or a neutron star into the companion star during the common envelope phase of close binary [3, 4]. The gravitational energy released in the disk can be very large, more than sufficient to stop the collapse of outer layers and drive GRB outflows, presumably in the polar direction where density is much lower [5]. GRB jets can be powered via heating due to annihilation of disk neutrinos [5] and via magnetic braking [6, 7, 8].

The high angular momentum assumed for the stellar core in the collapsar model also implies high rotation rates of the central black hole and the possibility of powering the GRB jets via the Blandford-Znajek mechanism [9]. The rotational energy of black hole with mass $M_{h}=$ $2 M_{\odot}$ and spin $a=0.9$ is enormous, $E_{\text {rot }} \simeq 5 \times 10^{53} \mathrm{erg}$, which is fifty times higher than the rotational energy of a millisecond pulsar [10] and well above what is needed to explain GRBs and associated hypernovae. Applying the Blandford-Znajek formula to the collapsar problem we obtain the following estimate of power

$$
\dot{E}_{B Z}=3.6 \times 10^{50} f(a) \Psi_{27}^{2} M_{2}^{-2} \mathrm{erg} \mathrm{s}^{-1},
$$

where $M_{2}=M_{h} / 2 M_{\odot}, \Psi_{27}=\Psi / 10^{27} \mathrm{Gcm}^{2}$ is the magnetic flux penetrating BH, $f(a)=a^{2} /\left(1+\sqrt{1-a^{2}}\right)^{2}$, and it is assumed that $\mathrm{BH}$ magnetosphere rotates with angular velocity $\Omega=0.5 \Omega_{h}$, thus delivering maximum power. These estimates show that the magnetic braking of BH could fully account for the energetics of GRBs. However, the result $\Omega=0.5 \Omega_{h}$ is obtained for force-free magnetospheres where one can ignore the inertia of matter and it is not clear when and how such conditions can develop in the collapsar model. For high mass-loading in the magnetosphere one could expect the magnetospheric rotation to be dictated by the angular momentum of matter rather then by the rotation of $\mathrm{BH}$ itself. Careful GRMHD simulations are needed in order to resolve this issue. Previous studies, with simplified setting and micro physics, have already demonstrated that this problem can be addressed with modern computational tools (e.g.[11]) and invite to explore progressively more realistic models. Here we present numerical solutions for models with realistic EOS and effects of neutrino cooling. The neutrino transport, however, is rather complicated, particularly in the curved space of $\mathrm{BH}$, and for this reason we have not included the heating due to neutrino annihilation. In reality, both the neutrino and magnetic mechanisms could operate hand in hand and we plan to include neutrino heating in future studies. The results for one particular numerical model have been presented in [1]; here we outline a wider study. The effects of neutrino cooling on disc accretion have been described in [12]. 


\section{SIMULATION SETUP}

Since our code can deal only with time-independent space-time we are forced to start from the point where the central BH of mass $M_{h}=3 M_{\odot}$ has already been formed inside the collapsing star. The rotation rate of $\mathrm{BH}$ is rather uncertain and depends on the angular momentum distribution in the progenitor. Here we use either the rather optimistic value of $a=0.9$ or the very pessimistic value of $a=0.0$. The latter was introduced to fully eliminate the Blandford-Znajek effect and to see if the magnetic braking of accretion disk alone could make the star to explode.

The main details of our numerical method and various test simulations are described in [13]. The only really new feature here is the introduction of HLL-solver which is activated when our linear Riemann solver fails, this occurs in regions of relativistically high magnetization. We have also found that total switch to the HLL-solver significantly degrades numerical solutions via increasing numerical diffusion and dissipation (see also [14]).

The gravitational attraction of $\mathrm{BH}$ is introduced via Kerr metric in Kerr-Schild coordinates, $\{t, \phi, r, \theta\}$. The two-dimensional computational domain is $\left(r_{0}<r<\right.$ $\left.r_{1}\right) \times(0<\theta<\pi)$, where $r_{0}=\left(1+0.5 \sqrt{1-a^{2}}\right) r_{g}$ and $r_{1}=5700 r_{g}=25000 \mathrm{~km}$.

Notice that the inner boundary is inside of the outer event horizon - we can do this because the horizon coordinate singularity is eradicated in the Kerr-Schild coordinates. The total mass within the domain is small compared to the mass of BH ( only about $7 \%$ ) that allows us to ignore its self-gravity. The grid is uniform in $\theta$ where it has 180 cells and almost uniform in $\log (r)$ where it has 450 cells, the linear cell size being the same in both directions.

A realistic equation of state (EOS) is introduced using the EOS code HELM" [15]. The neutrino cooling is computed using the interpolation formulas given in [16, [17, 18]. The photo-disintegration of nuclei is included via modifying EOS as in [19]. The equation for mass fraction of free nucleons is adopted from [20].

The collapsing star is described by the free-fall model of [21]. The collapse time for a core of radius $r_{c}=10^{9} \mathrm{~cm}$ and mass $3.0 M_{\odot}$ is $t_{c}=\sqrt{2} r_{c} / 3 \mathrm{v}_{\mathrm{ff}} \simeq 0.5 \mathrm{~s}$. Since, in this study we explore the possibility of explosion soon after the core collapse, we start simulations 1 second after the onset on collapse. Because GRBs are currently associated with very massive progenitors we set the mass parameter $C_{1}$ of Bethe's model (see [1] ) to either $C_{1}=$ 3 or $C_{1}=9$. These correspond to the accretion rates

\footnotetext{
1 the code can be downloaded from the website http://www.cococubed.com/code_pages/eos.shtml
}

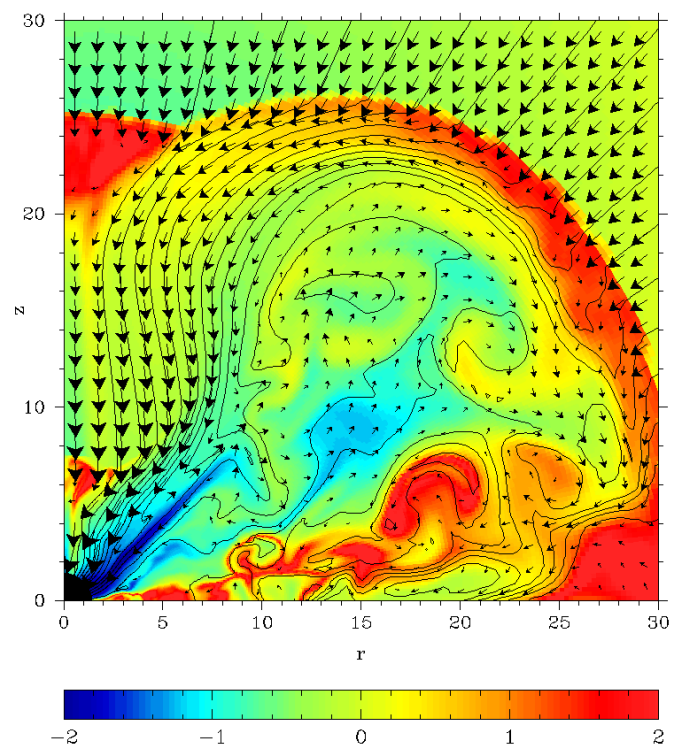

FIGURE 1. Model $C_{1}=9, B_{0}=3 \times 10^{10} \mathrm{G}$ at time $t=$ $0.24 \mathrm{~s}$, soon before the explosion. Colour shows the ratio of gas and magnetic pressure, $\log _{10}\left(P / P_{m}\right)$, contours the magnetic field lines and arrows the velocity field. Notice the outflow beginning to develop in the highly magnetized "blue stripe" stretching at the angle of $45^{\circ}$ from the black hole.

of $\dot{M} \simeq 0.166 M_{\odot} \mathrm{s}^{-1}$ and $\dot{M} \simeq 0.5 M_{\odot} \mathrm{s}^{-1}$ respectively. The angular momentum distribution describes a solid body rotation within the cylindrical $r_{l}=6300 \mathrm{~km}$ and is constant with the value of $l_{0}=10^{17} \mathrm{~cm}^{2} \mathrm{~s}^{-1}$ further out, similar to that used in [5]. These initial conditions are also used to fix the flow variables in the ghost cells of the outer boundary.

The magnetic field distribution is that of a uniformly magnetized sphere in vacuum, the radius of this sphere $r_{1}=4500 \mathrm{~km}$. Inside the sphere the magnetic field strength is $B_{0}=3 \times 10^{10} \mathrm{G}, 10^{10} \mathrm{G}$ or $3 \times 10^{9} \mathrm{G}$.

\section{COMPUTER SIMULATIONS}

At the beginning of simulations the angular momentum of gas near BH is less than that of the last stable orbit, $l_{l s o}$, and as a result it falls straight into the $\mathrm{BH}$. The most rapidly varying parameter at this stage is the magnetic flux threading the $\mathrm{BH}$ which grows linearly with time. Later, when matter with $l \simeq l_{l s o}$ reaches the BH it forms a thin inviscus accretion disk in the equatorial plane [22]. Finally, when the angular momentum of gas approaching the $\mathrm{BH}$ in the equatorial direction well exceeds $l_{l s o}$ the centrifugal force halts its infall and a turbulent accretion disk develops around the $\mathrm{BH}$. At the same time a strong accretion shock lifts from its surface. After passing the 

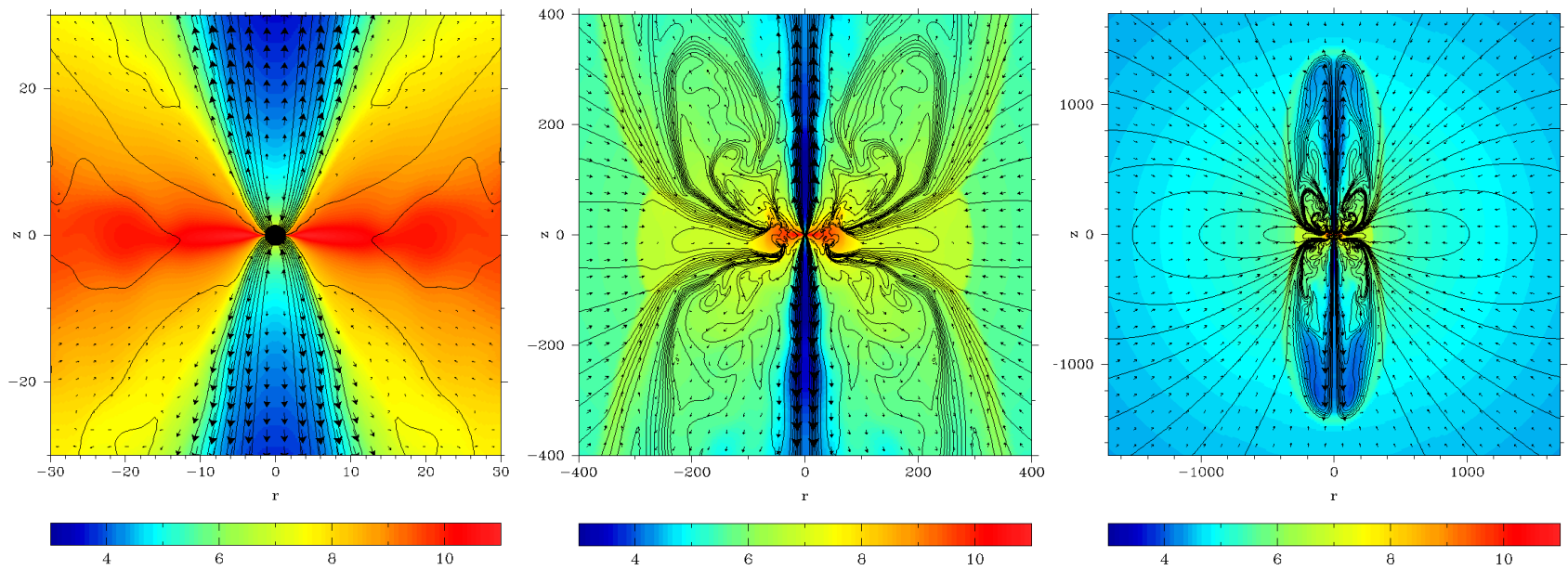

FIGURE 2. Solution for $C_{1}=3, B_{0}=10^{10} \mathrm{G}$ on different scales at $t=0.75 \mathrm{~s}$. Colour shows the baryonic rest mass density, $\log _{10} \rho$ in $\mathrm{g} / \mathrm{cm}^{3}$, contours the magnetic field lines, and arrows the velocity field.

accretion shock the low angular momentum plasma of polar regions keeps falling straight into the $\mathrm{BH}$ whereas the high angular momentum plasma coming at intermediate directions fills the low density volume above and below the disk. Strong differential rotation within this bubble results in strong amplification of azimuthal magnetic field. Further evolution depends of the amount of magnetic flux accumulated by the $\mathrm{BH}$.

In the model with $C_{1}=9$ and $B_{0}=3 \times 10^{10} \mathrm{G}$ we observed strong explosion very soon after formation of the accretion disk. The BH is a key factor in this explosion pumping the electromagnetic energy into the bubble at the rate of $\simeq 12 \times 10^{51} \mathrm{erg} \mathrm{s}^{-1}$. Prior to the explosion we see mass unloading of some magnetic field lines connecting the bubble with BH (see fig 11). This seems to be promoted by the fact plasma entering the accretion shock along these field lines is diverted towards the equatorial plane instead of entering the bubble and falling into the BH. Similar evolution was shown by the model described in [1]

In the model with $C_{1}=3$ and $B_{0}=10^{10} G$ we observe several pulsations of the accretion shock, gradual expansions followed by relatively rapid contractions, prior to the explosion which is significantly less energetic. However, once the explosion sets up it shows the same generic features (see fig 2). We observe two well defined polar jets surrounded by magnetic cocoons of high pressure and low density. The magnetic pressure of these cocoons, which have been inflated by the jets, exceeds by more than six orders of magnitude the magnetic pressure in the collapsing star. These over-pressured cocoons drive a blast wave into the star. The mean propagation speed of the blast in the polar direction $v_{s} \simeq 0.1 c$. In the vicinity of $\mathrm{BH}$ the solution shows the same key features as found in the previous studies of thick disks around $\mathrm{BHs}$ - main disk, its dynamic corona, and magnetically-dominated funnel inside which two Pointing-dominated jets are produced by the $\mathrm{BH}[23,24,25,1,26,12]$. Integrating over the volume of the blast wave we can measure the rate of energy supply in the explosion, $\dot{E} \simeq 0.4 \times 10^{51} \mathrm{erg} \mathrm{s}^{-1}$. The direct measurement of the energy flux across the horizon inside the funnel gives a very similar number, $\dot{E}_{B Z} \simeq 0.42 \times 10^{51} \mathrm{erg} \mathrm{s}^{-1}$, which is also in excellent agreement with eq.1. Thus, the explosion is undoubtedly powered by the Blandford-Znajek mechanism. Finally, this model seems to be marginal as any further noticeable increase of accretion rate or decrease of magnetic field strength prevents explosion.

Although initially the $\mathrm{BH}$ jets are Poynting-dominated their electromagnetic energy is gradually converted into the energy of matter. To some extent this may reflect real physical processes [11, 27, 28, 29] but numerical diffusion and resistivity are likely to play a significant part too. In any case, the total energy is conserved in the simulations and we do not expect these numerical problems to effect the dynamics of blast.

In order to investigate further the potential of magnetic breaking of accretion disk itself we carried out simulations with non-rotating BHs, thus eliminating the BZ-effect. The results show that the accretion disk is formed much later when almost all magnetic flux is already accreted onto the $\mathrm{BH}$ and it begins to "swallow" the closed magnetic field lines. As in other solutions, we observe lift-off of strong accretion shock, development of disk corona, and amplification azimuthal magnetic field. However, the explosion does not take place even under the most favourable conditions (the highest initial magnetic field and the lowest mass accretion rate) in our parameter space. This result provides an additional support to our conclusion on the crucial role of the BZ- 
mechanism.

Our results seem to fit the following condition for magnetically driven explosion

$$
\dot{M}_{0.1}^{-0.5} B_{10} r_{m, 9}^{2} \frac{a}{\left(1+\sqrt{1-a^{2}}\right)}>0.1,
$$

where $B_{0,10}=B_{0} / 10^{10} \mathrm{G}, r_{m, 9}=r_{m} / 10^{9} \mathrm{~cm}, \dot{M}_{0.1}=$ $\dot{M} / 0.1 M_{\odot} \mathrm{s}^{-1}$, which is obtained by comparing the maximum power of the BZ-mechanism (see eq.1) and the power of accretion flow. Further investigation is needed to verify this condition for intermediate values of the spin parameter. For $\dot{M}_{0.1}>0.5$ the power of neutrino annihilation becomes substantial and this may lead to a much less demanding criterion.

\section{DISCUSSION AND CONCLUSIONS}

Our results suggest that magnetic fields may play a crucial role in powering GRB jets and associated hypernovae not only in the magnetar but also in the collapsar model. In the latter case the main source of energy is the rotation of $\mathrm{BH}$ and it is released via the BlandfordZnajek mechanism. The rate of energy release seen in the simulations, $\dot{E}=0.2 \div 16 \times 10^{51} \mathrm{erg} \mathrm{s}^{-1}$, is very high and can easily explain the energetics of hypernovae, $E \simeq$ $10^{52} \mathrm{erg}$. The fact that the rotational energy of maximally rotating $\mathrm{BH}$ is much higher, $E_{\text {rot }} \simeq$ few $\times 10^{53} \mathrm{erg}$, suggests a self-regulating process in which the rate of energy release significantly reduces after the first few seconds, presumably due to the lower mass supply into the accretion disk when the stellar collapse is reversed by the blast, the escape of magnetic flux from the black hole, or its annihilation due to the accretion of magnetic field lines of opposite polarity. This idea is supported by the lower observed energetics of GRB jets. With the typical total energy of $10^{51} \mathrm{erg}$ and duration of $10 \div 100$ s their power is only $0.1 \div 0.01 \times 10^{51} \mathrm{erg} \mathrm{s}^{-1}$ [30, 31]. Alternatively, the hypernovae blast wave could be caused mainly by the neutrino heating during the short initial period of high accretion rate. In this case much weaker magnetic field will be required to drive GRB jets into the cavity created by the blast.

\section{ACKNOWLEDGMENTS}

This research was funded by PPARC under the rolling grant "Theoretical Astrophysics in Leeds". The simulations were carried out on the St Andrews UK MHD cluster and on the White Rose Grid cluster Everest.

\section{REFERENCES}

1. Barkov M.V., Komissarov S.S., Month. Not. Royal Astron. Soc.: Lett. 385, L28-L32 (2008).

2. Woosley S.E., Astrophysical Journal 405, 273-277 (1993).

3. Fryer C.L., Woosley S.E., Astrophysical Journal Lett. 502,L9-L12 (1998).

4. Zhang W., Fryer C.L., Astrophysical Journal 550, 357-367 (2001).

5. MacFadyen A.I. \& Woosley S.E., Astrophysical Journal 524, 262-289 (1999).

6. Blandford R.D., Payne D.G.,Month. Not. Royal Astron. Soc. 199, 883-903 (1982).

7. Uzdensky D.A. \& MacFadyen A.I., Astrophysical Journal 647, 1192-1212 (2006).

8. Proga D., MacFadyen A.I., Armitage P.J., Begelman M.C., Astrophysical Journal Lett. 599, L5-L8 (2003).

9. Blandford R.D. \& Znajek R.L., Month. Not. Royal Astron. Soc. 179, 433-456 (1977).

10. Komissarov S.S., Barkov M.V., Month. Not. Royal Astron. Soc. 382, 1029-1040 (2007).

11. McKinney J.C., Month. Not. Royal Astron. Soc. 368, 1561-1582 (2006).

12. Barkov M.V., preprint: astro-ph 0805.0414, 1-6 (2008).

13. Komissarov S.S., Month. Not. Royal Astron. Soc. 350, 1431-1436 (2004).

14. Mignone A., Bodo G.,Month. Not. Royal Astron. Soc. 368, 1040-1054 (2006).

15. Timmes F.X., Swesty F.D., Astrophysical Journal Supp. Series 126, 501-516 (2000).

16. Ivanova L.N., Imshennik V.S., Nadezhin D.K., Nauchnye Informatsii 13, 3 (1969).

17. Schinder P. J., Schramm D. N., Wiita P. J., et al., Astrophysical Journal 313, 531-542 (1987).

18. Bezchastnov V.G., Haensel P., et al., Astronomy and Astrophysics 328, 409-418 (1997).

19. Ardeljan N.V., Bisnovatyi-Kogan G.S.,et al., Month. Not. Royal Astron. Soc. 359, 333-344 (2005).

20. Woosley S.E., Baron E., Astrophysical Journal 391, 228-235 (1992).

21. Bethe H.A., Rev. Mod. Phys.,62, 801-866 (1990).

22. Beloborodov A.M., Illarionov A.F., Month. Not. Royal Astron. Soc. 323, 167-176 (2001).

23. De Villiers J.P., Hawley J.F., Krolik, J.H., Astrophysical Journal 599, 1238-1253 (2003).

24. McKinney J.C., Gammie C.F., Astrophysical Journal 611, 977-995 (2004).

25. Shibata M., Sekiguchi Yu, Takahashi R., Progress of Theor. Physics 118, 2, 257-302 (2007).

26. Stephens B.C., Shapiro S.L., Liu Y.T., Physical Review D 77,4,044001 (2008).

27. Komissarov S.S., Barkov M.V., Vlahakis N., Königl A., Month. Not. Royal Astron. Soc. 380, 51-70 (2007).

28. Barkov M.V., Komissarov S.S., preprint: astro-ph 0801.4861, 1-4 (2008).

29. Tchekhovskoy A., McKinney J.C., Narayan R., Month. Not. Royal Astron. Soc. 388, 551-572 (2008).

30. Bloom J.S., Frail D.A., Kulkarni S.R., Astrophysical Journal 594, 674-683 (2003).

31. Piran T., Reviews of Modern Physics 76, 1143-1210 (2005). 